\title{
COMBINED ADAPTIVE CONTROLLER FOR UAV GUIDANCE
}

\author{
B.R. Andrievsky, A.L. Fradkov \\ Institute for Problems of Mechanical Engineering \\ of Russian Academy of Sciences \\ 61, Bolshoy av., V.O., 199178 \\ Saint Petersburg, Russia \\ E-mail: $\{$ andr,alf $\} @$ control.ipme.ru; b_andri@yahoo.com
}

Keywords: Guidance, adaptive control, feedforward compensator.

\begin{abstract}
Combined adaptive control law for an autopilot of the unmanned aerial vehicle (UAV) homing guidance system is proposed. The adaptation algorithm provides prescribed attitude motion dynamics for different flight conditions. Simulation results verify efficiency of the combined adaptive controller in the case of significant uncertainty of the UAV parameters and time dependence of the guidance loop.
\end{abstract}

\section{Introduction}

The demands on modern guided systems are becoming more stringent than in the past. The unmanned aerial vehicle (UAV) autopilots have to be able to produce a response that is accurate and fast despite severe variations in speed and altitude of the airframe. Hence the challenge for autopilot design is to produce closed loop airframe responses that are much faster than the nature weathercock frequency of the airframe, and to produce accurate and fast response in the face of large parametric uncertainty [12]. The promising way to fulfill these requirements is application of the adaptive control technique. Chosen method of adaptation has to meet the conflicting requirements on the tuning rate and performance quality under the conditions of lack of the UAV state measurements. The variable-structure control technique, utilizing forced sliding motions ensures high adaptability in the some region of UAV parameters [11], but in the general case can not provide the optimal closed-loop system performance. The adaptation methods based on the $p a-$ rameter identification make the optimality easier to secure, but have relatively long period of tuning.

The combined adaptive controller [1-4, 8] uses both variablestructure and identification approaches and is able to meet the claims indicated above.

The so-called persistent excitation condition is well known condition of convergence of the parameter estimates to their true values [7, 10]. Fulfillment of this requirement is an open question for the considered system, because the UAV controlling input (the rudder deflection) is produced by the autopilot as a function on current UAV position and the command (reference) signal. This signal is produced for the autopilot by the guidance system and also depends on the UAV state variables. Besides, for practical applicability of the proposed scheme more realistic plant model (in comparison with $[1,5,9]$ ) has to be taken. First of all, it is necessary to take into account nonlinearities of the UAV model, atmospheric disturbances and sluggishness of the steering gears.

The considered homing guidance system is inherently nonlinear (due to nonlinearities in the adaptation/identification algorithms) and time-dependent (due to kinematics of approaching the target). Examination of the overall system is provided by means of the computer simulation.

\section{Combined adaptive controller}

The adaptation algorithm includes the bang-bang controller with forced sliding motion, parametric identification algorithm and the parallel feedforward compensator (or shunt [1, 5, 9]). The adaptation control law is an internal part of the subordinate control system. The main loop of the considered system is time-dependent. The control action in this loop can be considered as the reference signal for the internal adaptive controller. The adaptation algorithm provides the prescribed dynamic properties of the internal system when the plant parameters are changed in the wide range.

By means of the shunt [1] the UAV attitude angular velocities are not used in the control law.

The main loop of the considered system is used for approaching the target. The kinematic relations effect time-dependence of this system.

Let us consider the general equations of the LTI SISO continuous-time plant:

$$
\dot{x}_{p}(t)=A_{p} x_{p}(t)+B_{p} u(t), y_{p}(t)=C_{p} x_{p}(t),
$$

where $x_{p}(t) \in \mathbb{R}^{n}, u(t) \in \mathbb{R}, y_{p}(t) \in \mathbb{R}$. The plant transfer function can be written as:

$$
\mathrm{W}_{p}(s)=C_{p}\left(s I_{n}-A_{p}\right)^{-1} B_{p}=\frac{\mathrm{B}(s)}{\mathrm{A}(s)}
$$

where $s \in \mathbb{C}$ stands for the argument; $\operatorname{deg} \mathrm{A}(s)=n$, $\operatorname{deg} \mathrm{B}(s)=m, k=n-m$ is the plant relative degree. In the considered case of the UAV lateral motion, the model (1) 
can be written in the following form:

$$
\left\{\begin{array}{l}
\dot{\beta}(t)=\omega_{y}(t)+a_{z}^{\beta} \beta(t)-a_{z}^{\delta_{r}} \delta_{r}(t) \\
\dot{\omega}_{y}(t)=-a_{m_{y}}^{\beta} \beta(t)-a_{m_{y}} \omega_{y}(t)-a_{m_{y}}^{\delta_{r}} \delta_{r}(t) \\
\dot{\psi}(t)=\omega_{y}(t)
\end{array}\right.
$$

where $\psi(t), \omega_{y}(t)$ stand for the yawing angle and its velocity; $\beta(t)$ is an angle of slide; $\delta_{r}(t)$ is an angle of the rudder deflection; $a_{z}^{\beta}, a_{m_{y}}^{\beta}, a_{m_{y}}^{\omega_{y}}, a_{\beta}^{\delta}$ and $a_{m_{y}}^{\delta_{r}}$ are the UAV model parameters. Their values depend on the flight conditions and assumed to be a priory unknown. Let us also neglect the time lag of autopilot servo and consider the rudder deflection $\delta_{r}(t)$ as a control action. The yaw angle $\psi(t)$ only (without the yawing rate $\omega_{y}$ ) is assumed to be measurable by means of the UAV onboard sensors. Note, that Eq. (3) corresponds to the transfer function (2), where $\operatorname{deg} A(s)=3, \operatorname{deg} B(s)=1$ the relative degree $k=2$ and the matrices $A, B, C$ are as follows:

$$
A=\left[\begin{array}{ccc}
a_{z}^{\beta} & 1 & 0 \\
-a_{m_{y}}^{\beta} & -a_{m_{y}} & 0 \\
0 & 1 & 0
\end{array}\right], \quad B=\left[\begin{array}{c}
-a_{z}^{\delta_{r}} \\
-a_{m_{y}}^{\delta_{r}} \\
0
\end{array}\right]
$$

$C=[0,0,1]$.

\subsection{The identification algorithm}

To obtain the identification algorithm, let us rewrite the plant model (2) in the following form:

$$
\begin{aligned}
& y^{(n)}(t)+a_{1} y^{(n-1)}(t)+\cdots+a_{n} y(t) \\
& =b_{0} u^{m}(t)+\cdots+b_{m} u(t) .
\end{aligned}
$$

For the considered UAV model (3) it is valid: $a_{1}=a_{m_{y}}^{\omega_{y}}-a_{z}^{\beta}$, $a_{2}=a_{m_{y}}^{\beta}-a_{z}^{\beta} a_{m_{y}}^{\omega_{y}}, a_{3}=0 . b_{0}=-a_{m_{y}}^{\delta_{r}}, b_{1}=a_{m_{y}}^{\beta} a_{z}^{\delta_{r}}+$ $a_{m_{y}}^{\delta_{r}} a_{z}^{\beta}$, Equation (4) can be rewrtitten as follows:

$$
y^{(n)}=\varphi(t)^{T} \theta^{*}
$$

where the regressor $\varphi(t)=\left[-y^{(n-1)}, \ldots,-y, u^{(m)}, \ldots, u\right]^{T}$ and the vector of parameters $\theta^{*} \in \mathbb{R}^{n+m+1}$. Note, that for the considered system, because $a_{3}=0$, the number of unknown plant parameters can be reduced and $\theta^{*}$ can be taken as $\theta^{*}=$ $\left[a_{1}, a_{2}, b_{0}, b_{1}\right]^{T} \in \mathbb{R}^{4}$. Introducing the signals $\tilde{y}^{(n)}(t), \tilde{\varphi}(t)$ as

$$
D(p) \tilde{y}^{(n)}(t)=y(n)(t), \quad D(p) \tilde{u}(t)=u(t),
$$

where $p=d / d t ; D(s)=s^{n}+d_{1} s^{n-1}+\cdots+d_{n}$ is some Hurwitz polynomial, one gets the following equations:

$$
\tilde{y}^{(n)}=\tilde{\varphi}(t)^{T} \theta^{*}
$$

The signals $\tilde{y}(t), \tilde{\varphi}(t)$ can be produced by means of the filters:

$$
\begin{aligned}
& \dot{\xi}(t)=A_{d} \xi(t)+b_{d} y(t), \\
& \dot{\psi}(t)=A_{d} \psi(t)+b_{d} u(t),
\end{aligned}
$$

where $\xi(t), \psi(t) \in \mathbb{R}^{n} ; A_{d}, b_{d}$ have the phase space canonical form, $\operatorname{det}\left(s \mathbf{I}-A_{d}\right)=D(s)$. In the present study it is taken: $d_{1}=2 \omega_{f}, d_{2}=2 \omega_{f}^{2}, d_{3}=\omega_{f}^{3}$, where parameter $\omega_{f}>0$ is chosen bandwidth of the filters (7).
Let us consider now the fictitious system $\dot{\theta}^{*}(t)=w(t), \tilde{y}(t)=$ $\tilde{\varphi}(t)^{T} \theta^{*}(t)+v(t)$ with the state space vector $\theta^{*}$, output signal $\tilde{y}$, white noise disturbances $w(t), v(t)$, zero system matrix and the output matrix $\tilde{\varphi}(t)^{T}$. Applying the Kalman filtering technique to this system one gets the identification algorithm as follows:

$$
\begin{aligned}
& \dot{\theta}(t)=-\Gamma(t) \phi(t) \sigma(t), \\
& \dot{\Gamma}(t)=-\Gamma(t) \phi(t) \phi(t)^{T} \Gamma(t)+\alpha \Gamma(t),
\end{aligned}
$$

where $\theta(t) \in \mathbb{R}^{4}$ stands for the vector of the UAV model parameter estimates, $\theta=\left[\hat{a}_{1}, \hat{a}_{2}, \hat{b}_{0}, \hat{b}_{1}\right]^{T}$; $\phi(t)=\left[-\tilde{y}^{(2)},-\tilde{y}^{(1)}, \tilde{u}^{(1)}, \tilde{u}\right] \in \mathbb{R}^{4}$ is the regressor; $\alpha>0$ is the algorithm parameter.

\subsection{The sliding-mode controller}

The sliding-mode control law is used to ensure an ideal tracking of the UAV yawing angle $\psi(t)$ after the reference signal $r(t)$ and has a following form:

$$
\delta_{r}(t)=-k_{s} \sigma(t)-\gamma \operatorname{sign} \sigma(t),
$$

where $k_{s}, \gamma$ are the controller parameters, the tracking error $\sigma(t)$ is founded as $\sigma(t)=\bar{\psi}(t)-r(t)$. The signal $\bar{\psi}(t)$ is a sum of UAV yawing angle $\psi(t)$ and the shunt (parallel feedforward) output $y_{s}(t)$, i.e. $\bar{\psi}(t)=\psi(t)+y_{s}(t)$. The shunt transfer function [1] $W_{c}(s)=\frac{\kappa \varepsilon(\varepsilon s+1)^{k-2}}{(s+\lambda)^{k-1}}$ for the considered example turns into the function of the first-order inertial unit.

The reference signal $r(t)$ is produced by means of the tunable pre-filter to provide the prescribed dynamics of the inner closed-loop UAV attitude control system [1]. Pre-filter adjustment is produced on basis of parameter estimates $\theta(t)$. The input command signal of the pre-filter $\psi^{*}(t)$ is generated by means of the guidance system.

\subsection{The guidance law}

The proportional navigation guidance law is widely used for homing guidance systems [12]. In spite of this fact, in the present paper a pure pursuit method and a direct pointing method are used, because the focal point of the present study is examination of the efficiency of the combined adaptation law in the closed-loop guidance system. The guidance reference signal $\psi^{*}(t)$ for an adaptive autopilot has one of the following forms:

$$
\begin{aligned}
& \psi^{*}=-k_{g}\left(\psi-\Psi_{t}\right), \\
& \psi^{*}=-k_{g}\left(\Psi-\Psi_{t}\right),
\end{aligned}
$$

where $k_{g}$ is the guidance gain; $\Psi(t)=\psi(t)-\beta(t)$ is the UAV track angle; $\Psi_{t}$ is the azimuth angle of line-of-sight to a target (one can notice that $\psi-\Psi_{t}$ means the sighting angle to a target in the UAV body-frame axes). To describe the guidance 

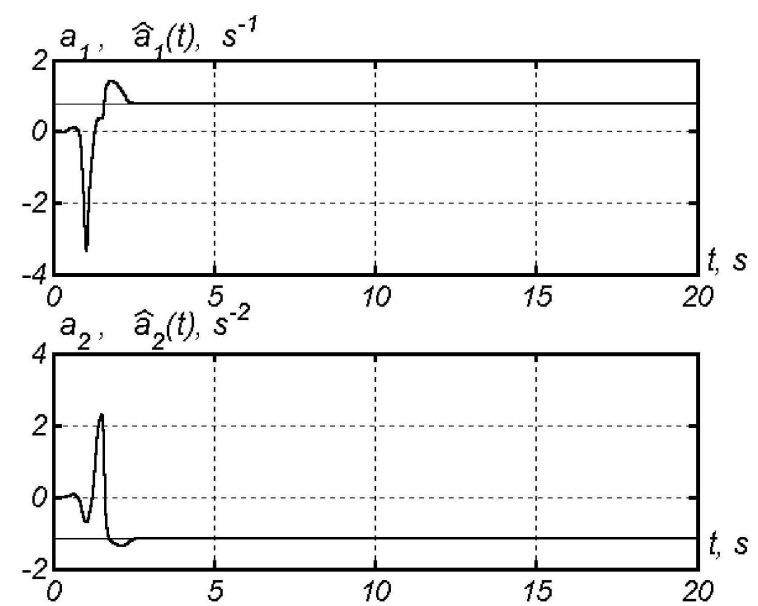

Figure 1: Time histories of the parameters $a_{1}, a_{2}$ estimates $\hat{a}_{1}(t), \hat{a}_{2}(t)($ Ex. \#1).
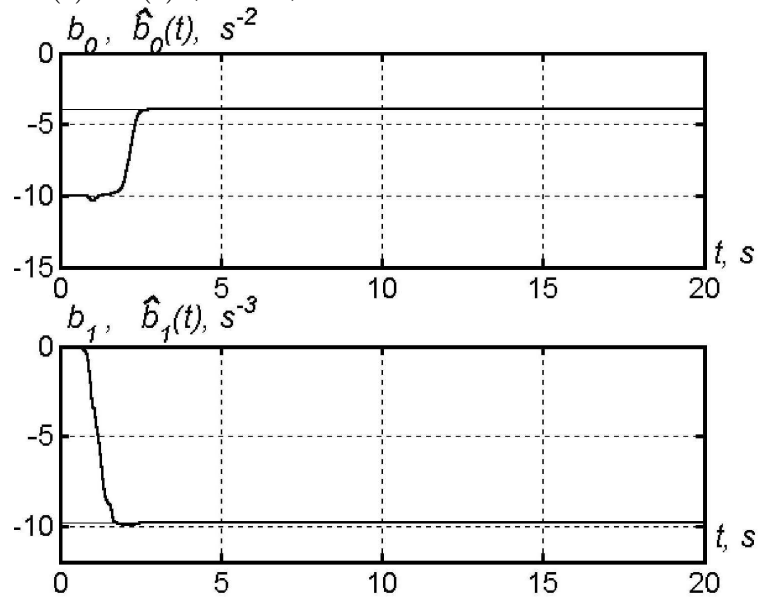

Figure 2: Time histories of the parameters $b_{0}, b_{1}$ estimates $\hat{b}_{0}(t), \hat{b}_{1}(t)($ Ex. \#1).

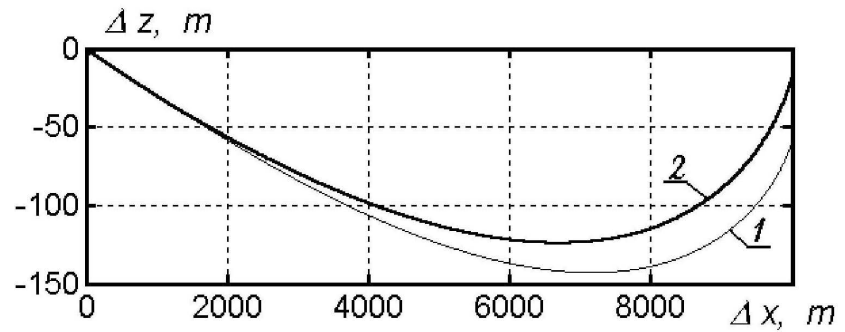

Figure 3: Flight path: 1 - guidance law (10); 2 - guidance law (11), (Ex. \#1).

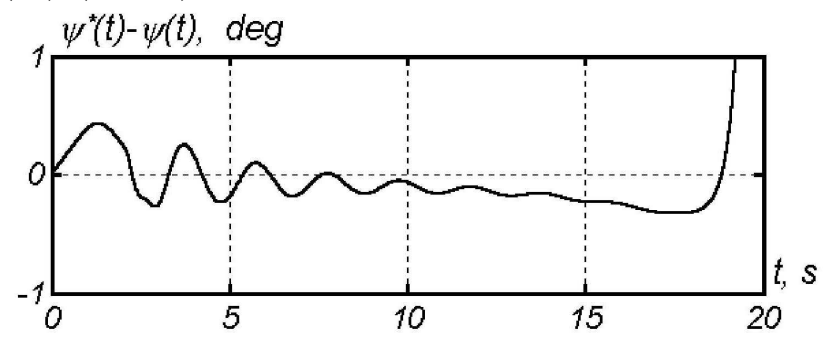

Figure 4: Tracking error $\Delta \psi(t)=\psi^{*}(t)-\psi(t)$ time history (Ex. \#1).

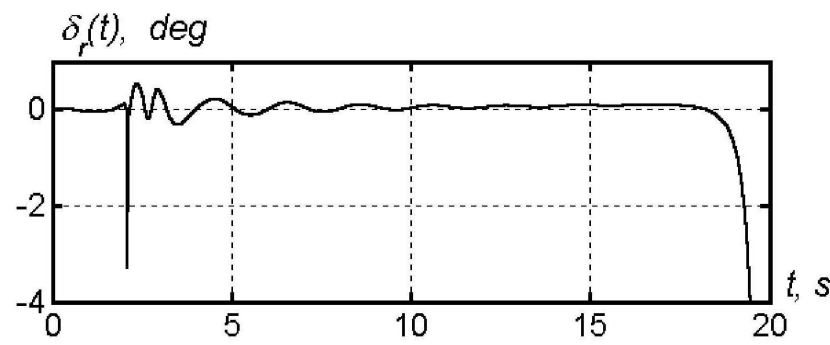

Figure 5: Rudder angle $\delta_{r}(t)$ time history (Ex. \#1).
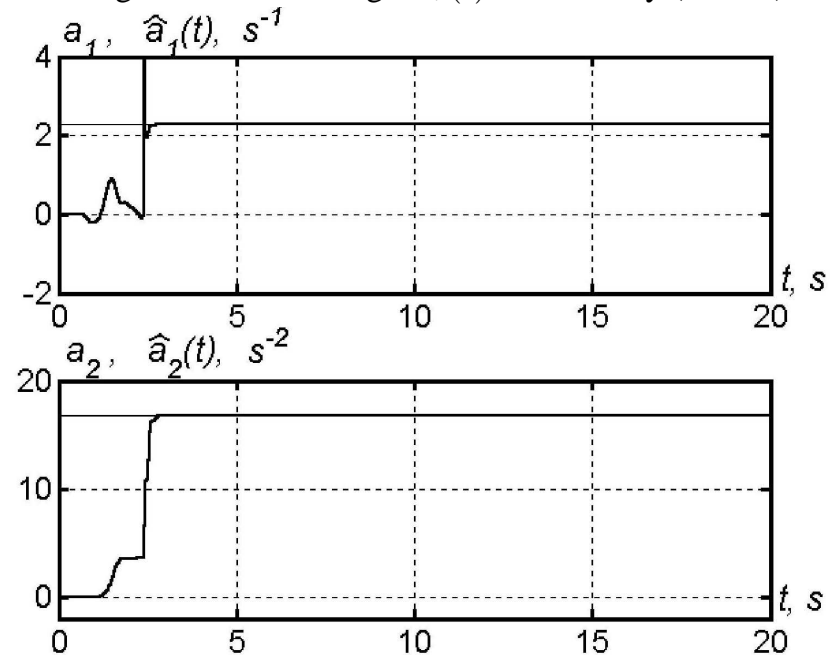

Figure 6: Time histories of the parameters $a_{1}, a_{2}$ estimates $\hat{a}_{1}(t), \hat{a}_{2}(t)($ Ex. \#2).
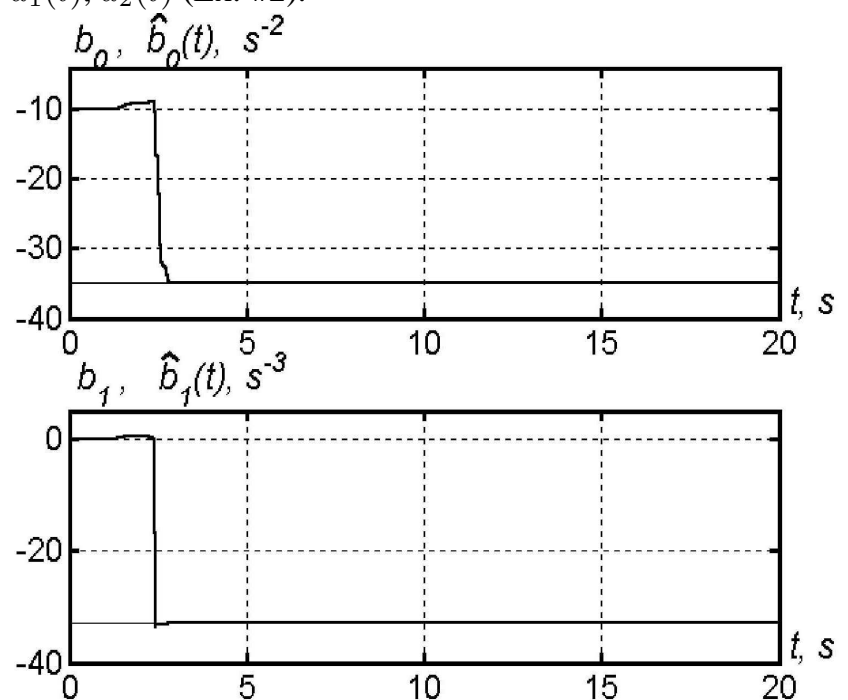

Figure 7: Time histories of the parameters $b_{0}, b_{1}$ estimates $\hat{b}_{0}(t), \hat{b}_{1}(t)$ (Ex. \#2).

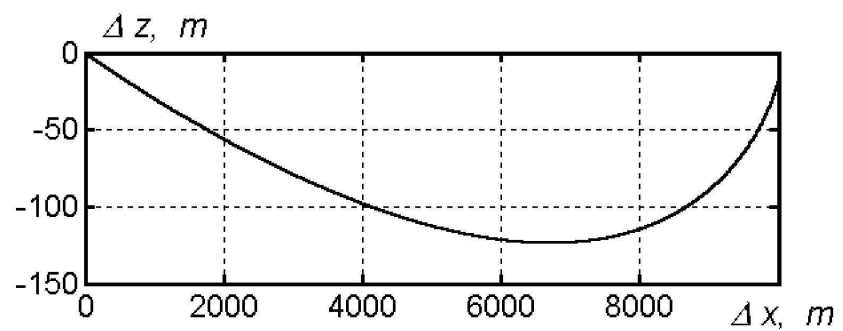

Figure 8: Flight path; the guidance law (11), (Ex. \#2). 
process, the following kinematic model is used:

$$
\begin{aligned}
& x=V t, \\
& x_{t}=D_{0}+V_{x_{t}} t, \quad z_{t}=z_{t 0}+V_{z_{t}} t, \\
& \Delta x=x_{t}-x, \quad \Delta z=z_{t}-z, \\
& D=\sqrt{\Delta x^{2}+\Delta z^{2}}, \\
& \dot{z}=-V \Psi, \\
& \Psi_{t}=-\arctan (\Delta z / \Delta x),
\end{aligned}
$$

where $V_{x_{t}}$ and $V_{z_{t}}$ are the components of the target velocity vector, so that $V_{x_{t}}=V_{t} \cos q_{t}, V_{z_{t}}=-V_{t} \sin q_{t} ; q_{t}$ is the target azimuth (in some Earth frame); $x(t), z(t), x_{t}(t), z_{t}(t)$ are, correspondingly, the UAV and target lateral coordinates; $V$ stands for the UAV ground speed.

\section{Numerical examples}

In this section some numerical examples and simulation results are presented.

Example 1. For this example the following parameters of the hypothetical UAV model have been taken: $a_{m_{y}}^{\beta}=-1.3 \mathrm{~s}^{-2}$, $a_{z}^{\beta}=-0.4 s^{-1}, a_{m_{y}}^{\delta_{r}}=9.85 s^{-2}, a_{z}^{\delta_{r}}=2.6 \cdot 10^{-3} s^{-1} a_{m_{y}}^{\omega_{y}}=$ $0.37 s^{-1}$ (one can notice that the unstable UAV model ith the set of eigenvalues $\{0,0.75,-1.53\}$ is presented). UAV ground speed $V=500 \mathrm{~m} / \mathrm{s}, V_{t}=20 \mathrm{~m} / \mathrm{s}, q_{t}=90 \mathrm{deg}$. Desired closed-loop attitude control system eigenvalues are picked up as follows: $s=\{-10,-2.1 \pm 2.14 i,-0.8\}$. Initial distance between UAV and target is taken equal to $10 \mathrm{~km}$.

The time histories of the UAV parameter estimates $\hat{a}_{1}(t), \hat{a}_{2}(t)$, $\hat{b}_{0}(t), \hat{b}_{1}(t)$ are shown in Figs. 1, 2. It can be seen, that the transient time of estimation processes is approximately $2.5 \mathrm{~s}$, and, therefore, the estimation rate is close to that one of the UAV attitude dynamics. In Fig. 3 the flight paths in the relative coordinates $(\Delta x, \Delta z)$ for two variants of the guidance law (10) and (11) are demonstrated. This results show that the law (11) for the case of the moving target is preferable. Figure 4 demonstrates the tracking error time history. It gives an idea of dynamic properties of the inner control loop. The rudder angle time history is shown in Fig. 5.

Example 2. Now let us consider the other flight conditions with the following parameter values of the UAV model: $a_{m_{y}}^{\beta}=$ $15.5 s^{-2}, a_{z}^{\beta}=-1.1 s^{-1}, a_{m_{y}}^{\delta_{r}}=33 s^{-2}, a_{z}^{\delta_{r}}=0.09 s^{-1}$ $a_{m_{y}}^{\omega_{y}}=1.2 s^{-1}$ This model parameters correspond to stable UAV attitude dynamics with the eigenvalues $\{0,-1.15 \pm$ $3.94 i\}$. The rest parameters of the UAV model, of the target motion, and of the control law are taken identical those ones in the previous example.

The simulation results for this example are shown in Figs. 610. These results demonstrate that the parameter estimates are obtained during the same time interval as for Ex. \#1. The flight path for this case is shown in Fig. 8. Obviously that it has neglible differences with that of Ex. \#1.

\section{Conclusions}

The combined adaptive control law for an autopilot of the UAV homing guidance system is proposed. The adaptive algorithm for UAV attitude control includes the variable-structure controller with forced sliding motion, parametric identification algorithm and the parallel feedforward compensator. The adaptation algorithm provides the prescribed dynamic properties of the attitude control system for various flight conditions of the UAV. Simulation results verify efficiency of the combined adaptive controller in the case of significant uncertainty of the UAV parameters and time-dependence of the main loop.

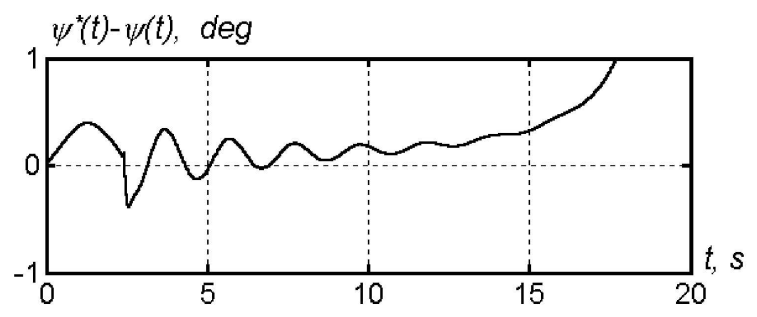

Figure 9: Tracking error $\Delta \psi(t)=\psi^{*}(t)-\psi(t)$ time history (Ex. \#2).

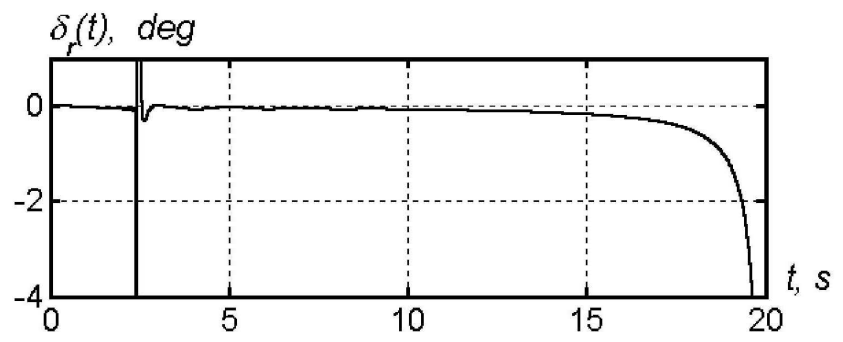

Figure 10: Rudder angle $\delta_{r}(t)$ time history (Ex. \#2).

\section{Acknowledgements}

The work was partly supported by Russian Foundation for Basic Research (grant 02-01-00765) and Presidium of Russian Academy of Sciences (scientific program 19, project 1.4).

\section{References}

[1] B.R. Andrievsky, A.L. Fradkov, A.A. Stotsky "Shunt compensation for indirect sliding-mode adaptive control", Proc. 13th Triennial IFAC World Congress, S. Fr., USA, (1996).

[2] B.R. Andrievsky, A.L. Fradkov. "Combined adaptive flight control system", Proc. 5th Intern. ESA Conference on Spacecraft Guidance, Navigation and Control Systems, Frascati, Italy, 22-25 October, (ESA-516, Feb. 2003), pp. 299-302, (2002).

[3] B.R. Andrievsky, A.L. Fradkov. "Combined adaptive autopilot for an UAV flight control”, Proc. 2002 IEEE International Conference on Control Applications, Glasgow, Scotland, U.K., pp. 290-291 (2002). 
[4] B.R. Andrievsky, A.L. Fradkov. "UAV guidance system with combined adaptive autopilot", Proc. IASTED International Conference on Intelligent Systems and Control, Salzburg, Austria, pp. 91-93 (2003).

[5] G. Bartolini, A. Ferrara, A. Stotsky. "Stability and Exponential stability of an adaptive control Scheme for plants of any relative degree", IEEE Trans. Autom. Contr. 40, No 1 , pp. $100-103,(1995)$.

[6] I. Bar-Kana. "Parallel feedforward and simplified adaptive control", Int. Journ. of Adapt. Control and Signal Processing. 1, pp. 95-109, (1987).

[7] A.L. Fradkov, I.V. Miroshnik, V.O. Nikiforov. "Nonlinear and Adaptive Control of Complex Systems", Dordrecht: Kluwer, (1999).

[8] A.L. Fradkov, B.R. Andrievsky. "Adaptive flight control based on parametric identification in the sliding mode", Proc. 9th Saint Petersburg International Conference on Integrated Navigation Systems, St.Petersburg, pp. 200202, (2002).

[9] Z. Iwai, I. Mizumoto. "Realization of simple adaptive control by using parallel feedforward compensator", Int. Journ. Contr., 59, pp. 1543 - 1565, (1994).

[10] K.S. Narendra, A.M. Annaswamy. "Persistant excitation in adaptive systems", Int. J. of Control, 45, 1, pp. 127160, (1987).

[11] V.I. Utkin. "Sliding Modes in Control and Optimization", Berlin: Sprienger-Verlag, (1992).

[12] B.A. White, A. Tsourdos "Modern missile flight control design: an overview", 15th IFAC Symposium on Automatic Control in Aerospace, Bologna, Italy, pp. 431-436, (2001). 